\title{
The Modifications of Wagner's Equation and Electrochemistry for the 21st Century
}

\section{Tomofumi Miyashita}

Miyashita Clinic, Osaka, Japan.

Email: tom_miya@ballade.plala.or.jp

Received January $4^{\text {th }}, 2011$; revised February $10^{\text {th }}, 2011$; accepted February $14^{\text {th }}, 2011$.

\begin{abstract}
The use of samarium-doped ceria (SDC) electrolytes in SOFCs (solid oxide fuel cells) lowers the open circuit voltage $(O C V)$ below the Nernst voltage $\left(V_{t h}\right)$. The OCV is calculated with Wagner's equation which is included in NernstPlanck equation. Considering the separation of Boltzmann distribution, the fundamental basis of this topic is discussed. A constant voltage loss without leakage currents due to a mixed ionic and electronic conducting (MIEC) dense anode was explained. Only carrier species having sufficient energy to overcome the activation energy can contribute to current conduction, which is determined by incorporating a different constant in the definitions of chemical potential and electrical potential. This difference explains the results using dense MIEC anodes. This topic is not an isolated and minor topic, but of vital importance to electrochemical engineering for the 21 st Century.
\end{abstract}

Keywords: SOFC, Ceria, Open Circuit Voltage, Mixed Ionic and Electronic Conductors, Wagner's Equation, Boltzmann's Distribution, Maxwell's Demon

\section{Introduction}

Solid-oxide fuel cells (SOFCs) directly convert the chemical energy of fuel gases, such as hydrogen or methane, into electrical energy. In SOFCs, a solid-oxide film is used as the electrolyte. Oxygen ions serve as the main charge carriers in the electrolyte. In these cells, YSZ (yttria-stabilized zirconia) is typically used as the electrolyte material. If the operating temperature (873-1273 K) were lowered, the lifespan of the cells would be extended. Lowering the temperature enables the use of higher ion-conducting electrolyte materials, such as Sm-doped ceria electrolytes (SDC).

However, the open current voltage (OCV) using an $\mathrm{SDC}$ cell is about $0.8 \mathrm{~V}$, which is lower than the Nernst voltage $\left(V_{t h}=1.15 \mathrm{~V}\right)$ at $1073 \mathrm{~K}$. This low OCV value is considered to be due to the low value of the ionic transference number $\left(t_{i o n}\right)$. OCV can be explained by Wagner's equation [1];

$$
\begin{gathered}
O C V=t_{i o n} \times \frac{R T}{4 F} \ln \left(\frac{p O_{2}^{\prime}}{p O_{2}^{\prime \prime}}\right)=t_{i o n} \times V_{\text {th }} \\
t_{\text {ion }}=\frac{R_{e}}{R_{i}+R_{e}}
\end{gathered}
$$

where $\mathrm{R}, \mathrm{T}$ and $\mathrm{F}$ are the gas constant, the absolute temperature in Kelvin and the Faraday constant, $p O_{2}^{\prime}$ and $p O_{2}^{\prime \prime}$ are the oxygen partial pressures at the cathode and anode, respectively, and $R_{i}$ and $R_{e}$ are the ionic resistance and the electronic resistance of the electrolyte, respectively. In general, tion is not constant in the electrolyte. Therefore, Wagner's equation is expressed as [2];

$$
O C V=\frac{R T}{4 F} \int_{p O_{2}^{\prime}}^{p O_{2}^{\prime \prime}} t_{\text {ion }} d \ln p O_{2}
$$

In Equation (3), the experimental verification of leakage currents is necessary $[3,4]$. Furthermore, theoretical limitation of Equation (3) was discovered [5]. The constant voltage loss without leakage currents due to a mixed ionic and electronic conducting (MIEC) dense anode is proposed with empirical equation. Furthermore, the modifications of Wagner's equation are proposed. This topic is not an isolated and minor topic, but of vital importance to electrochemical engineering for the 21st Century. Several future technological applications are also introduced.

\section{Experimental}

The SDC electrolyte was $25 \mathrm{~mm}$ in diameter and 600 $\mathrm{mm}$ thick. Porous Pt electrode (10 $\mathrm{mm}$ in diameter) was 
attached as a cathode electrode. Ni-YSZ (5:5 using volume ratio) cerrmet was used as an anode electrode. Ni particle size was $5 \mu \mathrm{m}$. YSZ was $80 \mu \mathrm{m}$ and sintered at $1400^{\circ} \mathrm{C}$ for 5 hours. Oxygen gas was fed to the cathode, and hydrogen gas with 3\% steam was supplied to the anode as the fuel gas. The operating temperature was $1073 \mathrm{~K}$. The time dependence of the output voltage was measured with $200 \mathrm{~mA}$ external current (current density $255 \mathrm{~mA} / \mathrm{cm}^{2}$ ) in two hours. During measurement, this current was stopped in 20 minutes.

\section{Results and Discussion}

When the external current was $200 \mathrm{~mA}$, output voltage was decreased during the measuring time. When the external current was stopped, output voltage $(=\mathrm{OCV})$ was unchanged, as shown in Figure 1 [3].

\subsection{The Modification of Wagner's Equation}

\subsubsection{Experimental Verification of Wagner's Equation}

The equation for OCV using SDC electrolytes is,

$$
\mathrm{OCV}=V_{t h}-R_{i} I_{i} \text { - polarization voltage losses. }
$$

Here Ii is ionic current. $R_{i}$ is constant, but polarization voltage losses should be changed with time, since every electrode worsens with time. So, the value of OCV using SDC electrolytes should be changed. Consequently, Wagner's equation was denied experimentally. The unchanged OCV was discovered in 1995 and published at PcRim2 (Cairns in Australia) in 1996. The detail mathematical calculation was published in 2006 [3]. Since 1996, the unchanged OCV has never been denied experimentally. Furthermore, numerous efforts have been

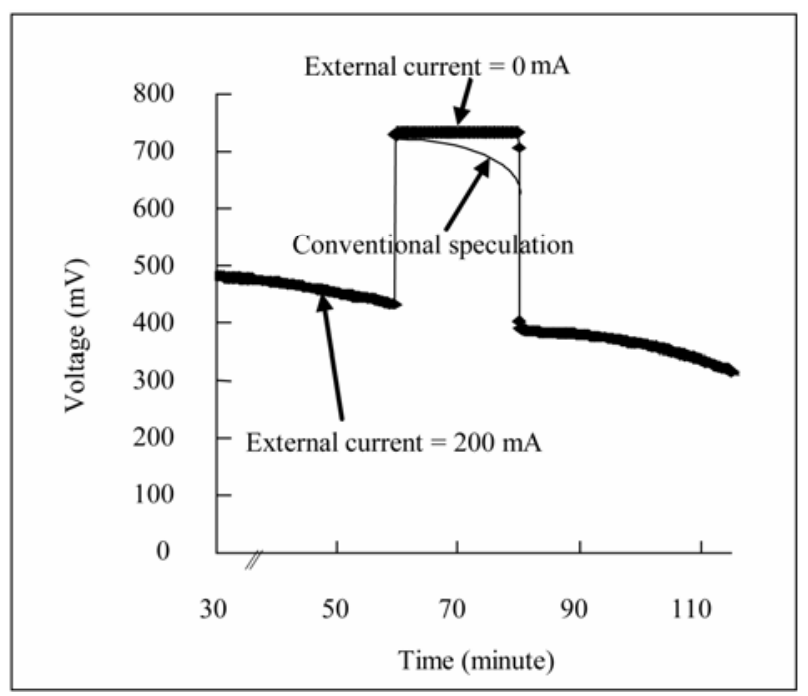

Figure 1. OCV was $0.75 \mathrm{~V}$ unchanged with rapidly aggregating anode that differed from theoretical results. made to solve the basic transport equation that describes SOFCs with MIECs. But current - voltage relationship considering electrode degradation has never been successful [4].

\subsubsection{Theoretical Limitation of Wagner's Equation}

From Ohm's law,

$$
\begin{gathered}
E_{\text {ions }}=\frac{I_{i}}{\sigma_{i} S} \\
E_{\text {electrons }}=\frac{I_{e}}{\sigma_{e} S}
\end{gathered}
$$

where $E_{\text {ions }}, E_{\text {electrons }}, \sigma_{i}, \sigma_{e}$ and $S$ are the electric field for ions, electric field for electrons, ionic conductivity, electronic conductivity and the cross-section of the electrolyte, respectively. When tion is not constant in Equation (3), $E_{\text {electrons }}$ and $\sigma_{e}$ cannot be constant. $E_{\text {electrons }}$ using $0.02 \mathrm{~cm}$ thickness SDC electrolyte at 873 $\mathrm{K}$ near the cathode is greater than $800 \mathrm{kV} / \mathrm{mm}$. This value is large enough to cause dielectric breakdown, which has never been reported, as 1-mm-thick Pyrex glass undergoes dielectric breakdown at only $20 \mathrm{kV}$ [5].

\subsubsection{Empirical Equation about OCV}

Instead of Wagner's equation, empirical equation was discovered. When the tion is almost unity at the cathode side and tion is small enough at the anode side, the new equation for the $\mathrm{OCV}$ is expressed as [6];

$$
\mathrm{OCV}=V_{t h}-E_{a} / 2 \mathrm{e} .
$$

$E_{a}$ is the activation energy of the oxygen ions and material constant parameter. The examples of Equation (7) are shown in Table 1. $E_{a}$ was measured from Arrhenius' plots which were matched with the public data.

\subsubsection{Macroscopic Explanation about the Modifications of Wagner's Equation}

Considering the separation of Boltzmann distribution, the fundamental basis of this topic is explained. The Boltzmann distribution of oxygen ions is shown in Figure 2. Ions with an energy over the ionic activation energy become carriers that can escape from the electrolyte. Since the Boltzmann distribution cannot be separated using passive filters, a problem known as the "Maxwell's demon," the distribution in Figure $\mathbf{3}$ is forbidden.

The electrochemical potential should be identical between carriers and non-carriers.

$$
\eta_{i_{-} \text {hopping }}=\eta_{i_{-} \text {vacancy }}
$$

Here, $\eta_{i \_ \text {hopping }}$ and $\eta_{i \_ \text {vacancy }}$ are the electrochemical potential of hopping ions and ions in vacancies, respectively. Therefore the correct distribution of hopping ions 
Table 1. Example of empirical equation ( $V_{t h}$ is $1.15 \mathrm{~V}$ at $1073 \mathrm{~K}$ and $1.18 \mathrm{~V}$ at $\left.873 \mathrm{~K}\right)$.

\begin{tabular}{cccc}
\hline Material & Measured Ea & Measured OCV & Calculated OCV \\
\hline $\mathrm{Sm}_{2} \mathrm{O}_{3}$ doped $\mathrm{CeO}_{2}$ & $0.68 \mathrm{eV}$ & $0.81 \mathrm{~V}$ & $0.81 \mathrm{~V}=1.15-0.68 / 2$ \\
$\mathrm{Sm}_{2} \mathrm{O}_{3}$ doped $\mathrm{CeO}_{2}$ & $0.68 \mathrm{eV}$ & $0.84 \mathrm{~V}$ & $0.84 \mathrm{~V}=1.18-0.68 / 2$ \\
$\mathrm{Gd}_{2} \mathrm{O}_{3}$ doped $\mathrm{CeO}_{2}$ & $0.74 \mathrm{eV}$ & $0.78 \mathrm{~V}$ & $0.78 \mathrm{~V}=1.15-0.74 / 2$ \\
$\mathrm{CaO}$ doped $\mathrm{CeO}_{2}$ & $0.83 \mathrm{eV}$ & $0.74 \mathrm{~V}$ & $0.74 \mathrm{~V}=1.15-0.74 / 2$ \\
$\mathrm{BaCeO}_{3}$ & $0.65 \mathrm{eV}$ & $1.1 \mathrm{~V}$ & $0.63 \mathrm{~V}=1.18-1.1 / / 2$ \\
$\mathrm{Er}_{2} \mathrm{O}_{3}$ doped $\mathrm{Bi}_{2} \mathrm{O}_{3}$ & About $1.0 \mathrm{eV}$ & $0.6 \mathrm{~V}-0.7 \mathrm{~V}$ & $0.68 \mathrm{~V}=1.18-1.0 / 2$ \\
\hline
\end{tabular}

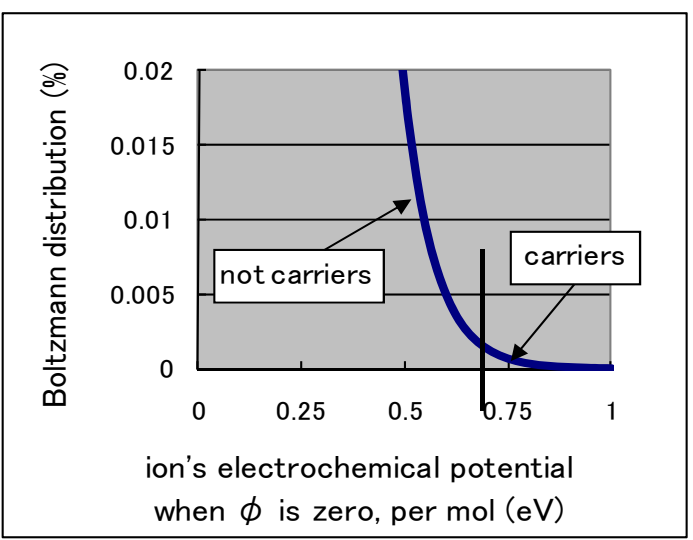

Figure 2. Boltzmann distribution of ion's electrochemical potential.

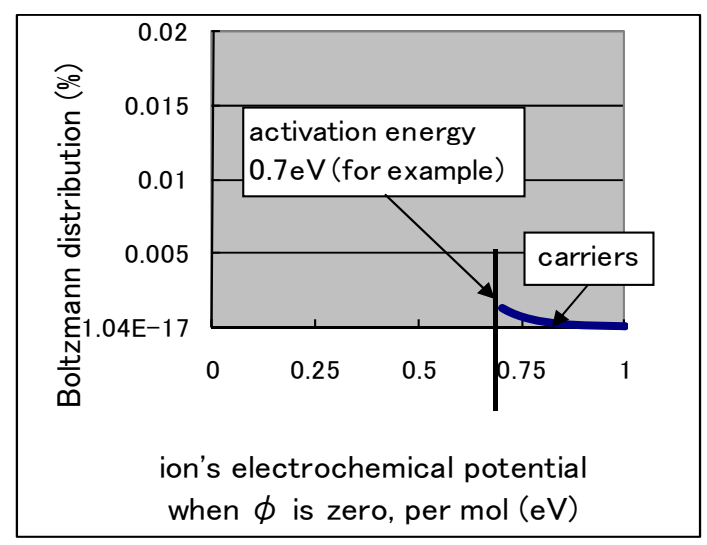

Figure 3. Incorrect distribution of hopping ions.

is shown in Figure 4. From the conventional theory,

$$
\eta_{i_{-} \text {hopping }}=\eta_{i_{-} \text {vacancy }}+N E_{a}
$$

Here, $N$ and $E_{a}$ are Avogadro's number and the activation energy of the oxygen ions, respectively. Equation (9) is different from Equation (8). Then, next transformations are needed.

$$
\begin{gathered}
\mu_{i \_ \text {hopping }}=\mu_{i \_ \text {vacancy }}+N E_{a} \\
Z F \phi_{\text {hopping }}=Z F \phi_{\text {vacancy }}-N E_{a}
\end{gathered}
$$

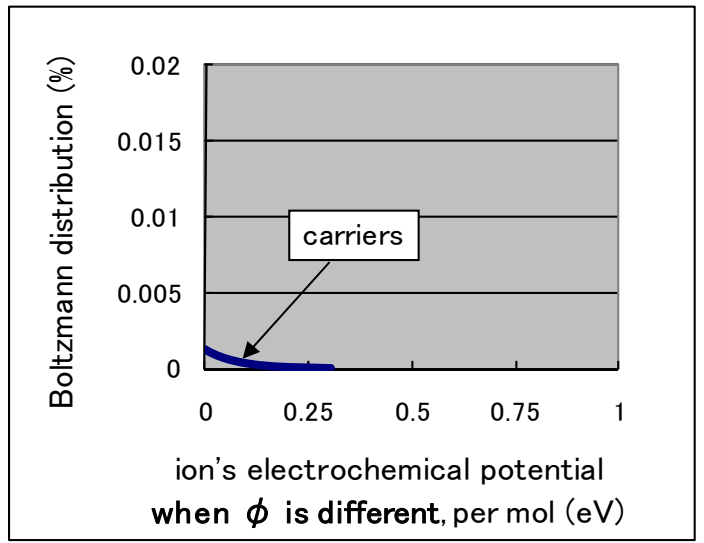

Figure 4. Correct distribution of hopping ions.

where $\mu_{i \_ \text {hopping, }}, \mu_{i_{-} \text {vacancy }}, \phi_{\text {hopping }}$ and, $\phi_{\text {vacancy }}$ are the chemical potential of the hopping ions, that of the ions in vacancies, the electrical potential of hopping ions and the electrical potential of ions in vacancies, respectively. Equation (10) and Equation (11) are the modifications of Wagner's equation.

From Equation (11), including $(Z=-2, N / F=1 / e)$,

$$
\phi_{\text {hopping }}=\phi_{\text {vacancy }}+E_{a} / 2 e
$$

In MIEC materials, $\phi_{\text {hopping }}$ is neutralized by free electrons. So,

$$
\mathrm{OCV}=\mathrm{V}_{\mathrm{th}}-E_{a} / 2 e
$$

Consequently, Equation (13) (= Equation (7)) can be explained by Equation (10) and Equation (11) which are the modifications of Wagner's equation.

\subsubsection{Microscopic Explanation about the \\ Modifications of Wagner's Equation}

Considering hopping mechanism, Equation (10) and Equation (11) can be explained. Using pure ionic conductors, a schematic view is shown in Figure 5. $\phi_{\text {hopping }}$ is not $\phi_{\text {vacancy }}$, since hopping ions at the saddle point are surrounded by cations in the lattice structure. It means that electrical potential energy transfers to lattice structure at 
the saddle point and send back to ions just after hopping.

Using MIEC materials, free electrons get the potential energy of ions at the saddle point. So, $\phi_{\text {hopping }}$ is neutralized by free electrons and the electrical potential energy cannot be sent back to ions. A schematic view is shown in Figure 6. The energy loss is $E_{a}$ and the voltage loss is $E_{a} / 2 e$. So,

$$
\mathrm{OCV}=V_{t h}-E_{a} / 2 \mathrm{e}
$$

\subsubsection{The Modification Necessity of Nernst Plank Equation for the 21st Century}

About the separation of Boltzmann's distribution using passive filter, the problem is known as "Maxwell's demon" which was already solved by Rolf William Landauer in 1961. This problem was solved by information theory, but the effects in the electrochemistry have never been shown. The effects are observed using MIEC. Not only Wagner's equation, but also Nernst-Plank equation should be modified, since Wagner's equation is based on the Nernst-Plank equation.

Thermally excited ions

$\mu_{i_{-} \text {hopping }}=\mu_{i_{-} \text {vacancy }}+N E_{a}$
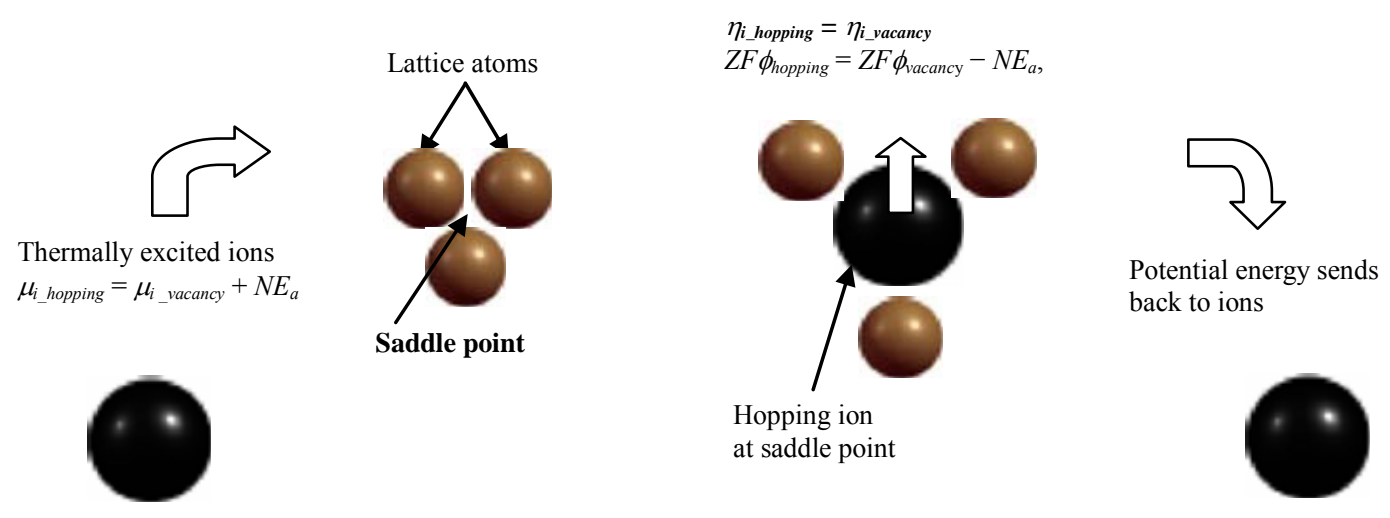

\subsection{Electrochemistry for the 21st Century}

\subsubsection{The Voltage Measurement Using MIEC}

In Figure 7, the voltage between 1 and 5 can become lower than the theoretical values when the anode part becomes MIEC. Without MIEC knowledge, it is impossible to clarify the mechanism of the possible voltage loss. Deviations of the experimental voltage from the theoretical voltage can only be attributed to the following explanations:

1) There are leakage currents.

2) The ion concentrations are different from expected values.

3) There is contamination from unknown ions.

In order to clarify the mechanism of voltage loss, the following statement must hold:

"The voltage loss is caused by the MIEC dense layer without any leakage currents."

\subsubsection{Theoretical Efficiency Using MIEC}

Ozone is produced in the $1.25 \mathrm{~mm}$ gap from many elec-

Figure 5. Schematic view of a different constant in the definitions of chemical potential and electrical potential during hopping.

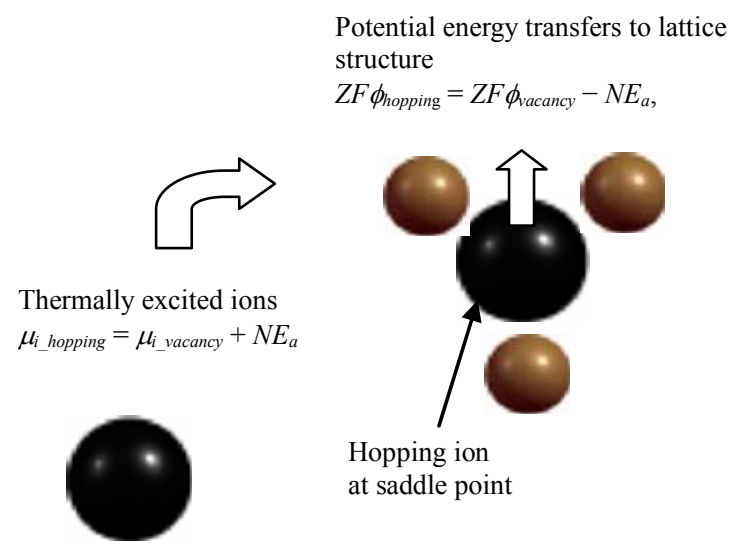

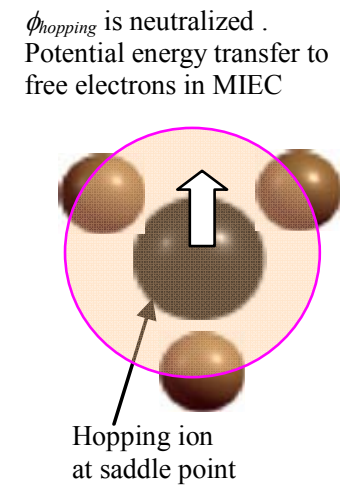

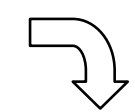

Free electrons get energy from ions

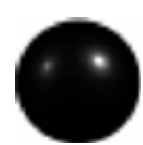

Figure 6. Schematic view of potential energy loss of ions in MIEC. 
trical discharges reacting with oxygen gas shown in Figure 8:

$$
\begin{gathered}
\mathrm{O}_{2}+\text { electron (electrical discharges) } \rightarrow 2 \mathrm{O} \\
\mathrm{O}+\mathrm{O}_{2} \rightarrow \mathrm{O}_{3}
\end{gathered}
$$

The technological efficiency is only $7 \%$ of the theo-

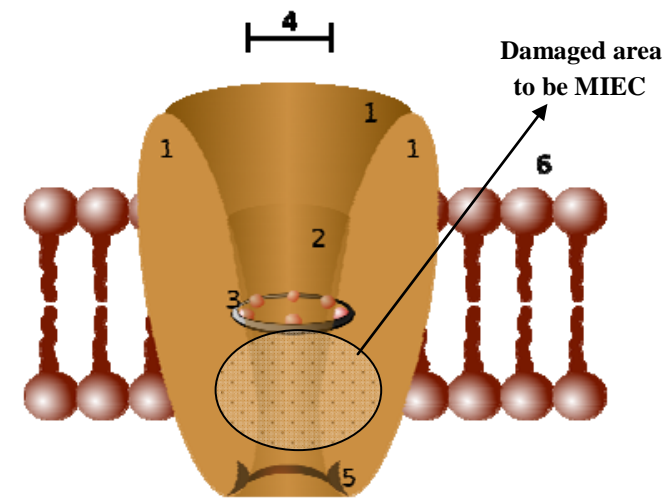

Figure 7. Schematic diagram of an ion channel (from free Wikipedia, http://en.wikipedia.org/wiki/Ion_channel) 1: Channel domains (typically four per channel), 2: Outer vestibule, 3: Selectivity filter, 4: Diameter of selectivity filter, 5: Phosphorylation site, 6: Cell membrane.

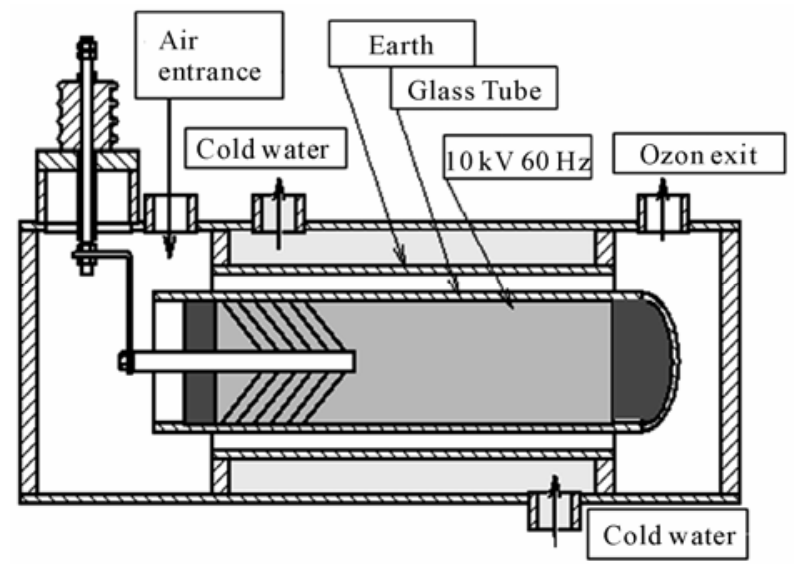

Figure 8. The structure of ozone generator.

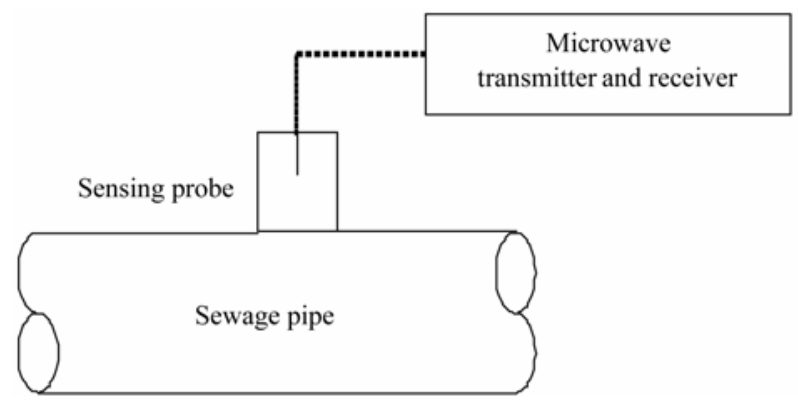

Figure 9. Sludge concentration measurement using microwave.

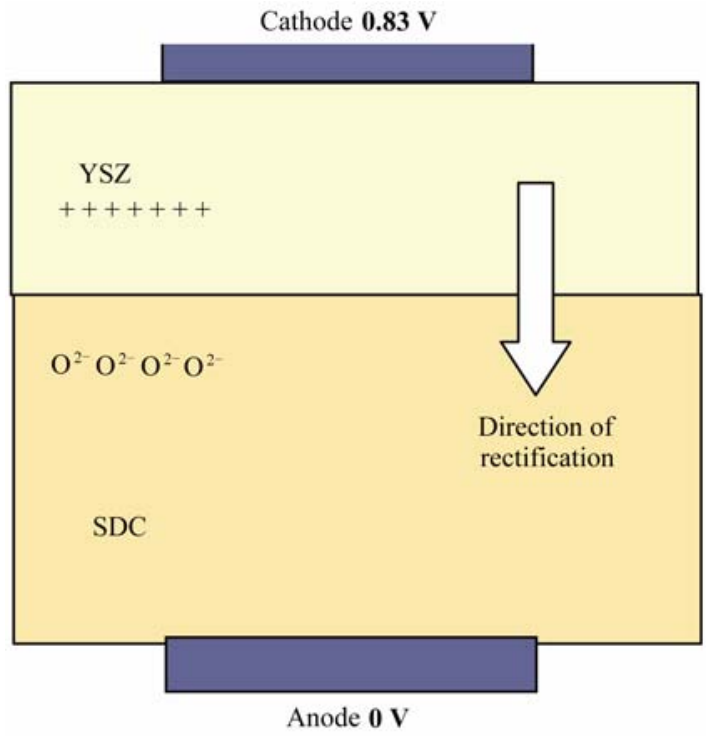

Figure 10. OCV is larger than a single SDC.

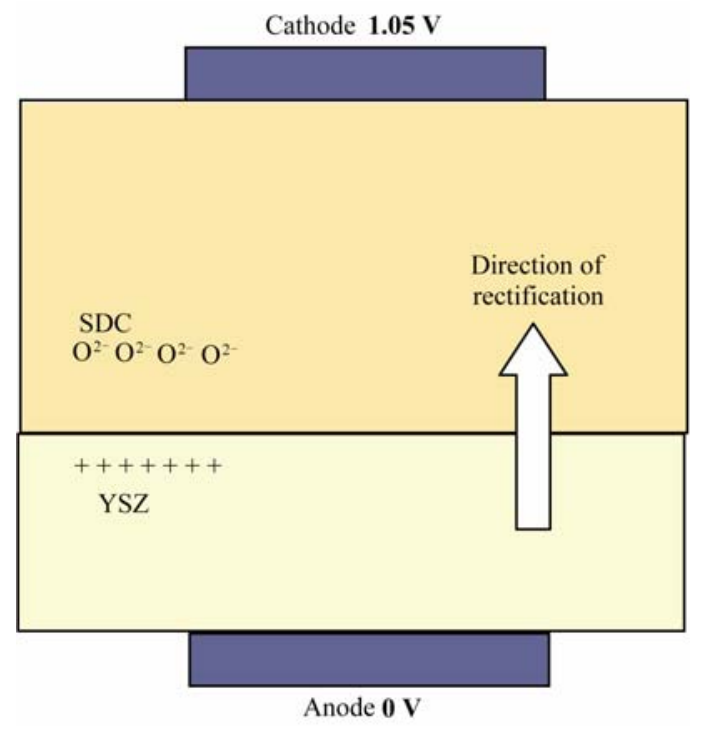

Figure11. OCV is smaller than Nernst voltage.

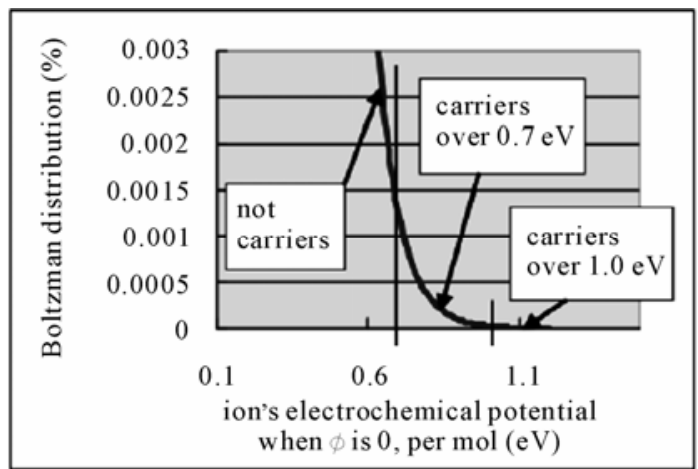

Figure 12. Explanation of rectification between two electrolytes. 

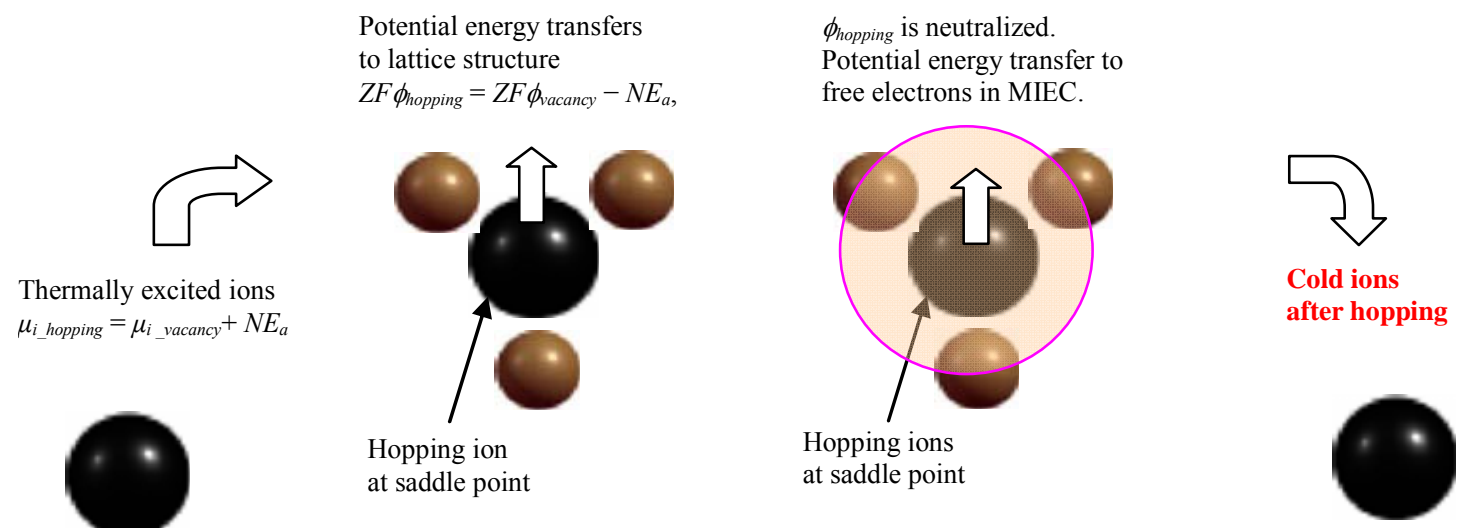

Figure 13. Explanation of cold ions after hopping.

retical efficiency. In 1990s, the research to improve efficiency had been done. As a result, many engineers thought that the low efficiency was not caused from technological reasons, but from unknown errors in the calculation of theoretical efficiency. Electrical discharges are MIEC. Without MIEC knowledge, it is impossible to clarify the low efficiency.

\subsubsection{The Power Loss Measurement Using MIEC}

Sludge concentration using microwave shown in Figure $\mathbf{9}$ is measured by next equation.

Sludge concentration $=$ power loss $($ in clean water $)-$ power loss (in dirty water)

This equation can be used only when the conductivity of water is small. In the power loss calculation, there need the compensation both for temperature and conductivity. The compensation for temperature is changed when the conductivity is changed. Dirty water is MIEC. Without MIEC knowledge, it is impossible to calculate the power loss.

\subsubsection{Water Electrolysis and Rectification}

Water electrolysis may be more important than SOFCs. Producing cheap hydrogen gas is an important future goal; however, these technologies are years from practical application. Rectification between two oxides must be solved. In Figure 10, OCV is larger than using a single SDC electrolyte. In Figure 11, OCV is smaller than Nernst voltage. When there are no leakage currents in a single SDC electrolyte, there should be rectification. The distribution of non-carriers is different in different ionic electrolytes, which explains the rectification shown in Figure 12. Without MIEC knowledge, it is impossible to notice the existence of rectification.

\subsubsection{Super Conducting Oxides}

In MIEC materials, ions lose energy after traveling the saddle point, since free electrons gain energy. The value of energy loss during one hop is equal to the ionic activation energy. It means that ions can be colder than electrolytes (for example $93 \mathrm{~K}$ ). The situation is shown in Figure 13. If hopping ions are cold enough (for example $1 \mathrm{~K}$ ), according to BCS theory, they can be the centers of superconductivity.

\subsubsection{The Research of the Consciousness}

According to Roger Penrose, the research of the consciousness should be the result of quantum effects, since it cannot be explained by the classical thermodynamics.

Table 2. The differences between brain and SDC.

\begin{tabular}{ccccc}
\hline & Structure & Arrangement & Symmetry & $\begin{array}{c}\text { Ionic activation } \\
\text { energy }\end{array}$ \\
\hline SDC & lattice & highly order & $\begin{array}{c}\text { Many } \\
\text { directions }\end{array}$ & Only one \\
Brain & fractal & highly order & none & $\begin{array}{c}\text { Number of } \\
\text { ion channels }\end{array}$ \\
\hline
\end{tabular}

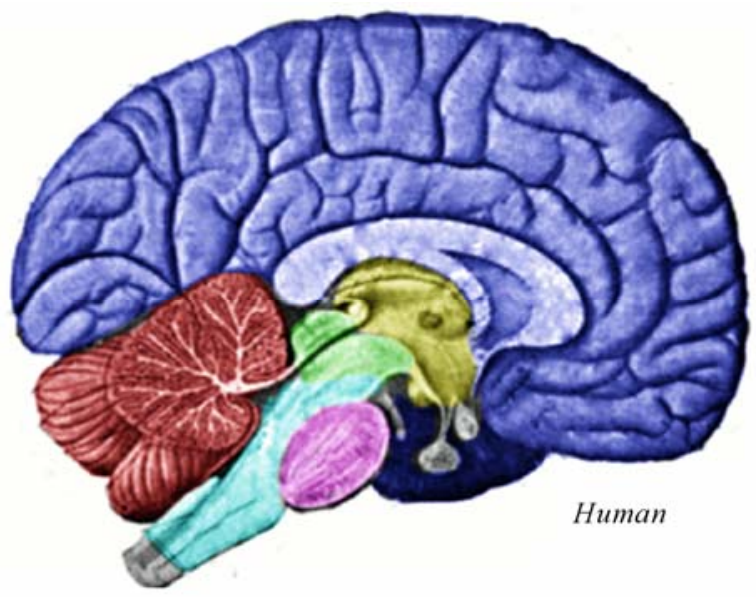

Figure 14. The structure of brain is fractal and different from the lattice structure (from http://en.wikipedia.org/wiki/ Brain). 


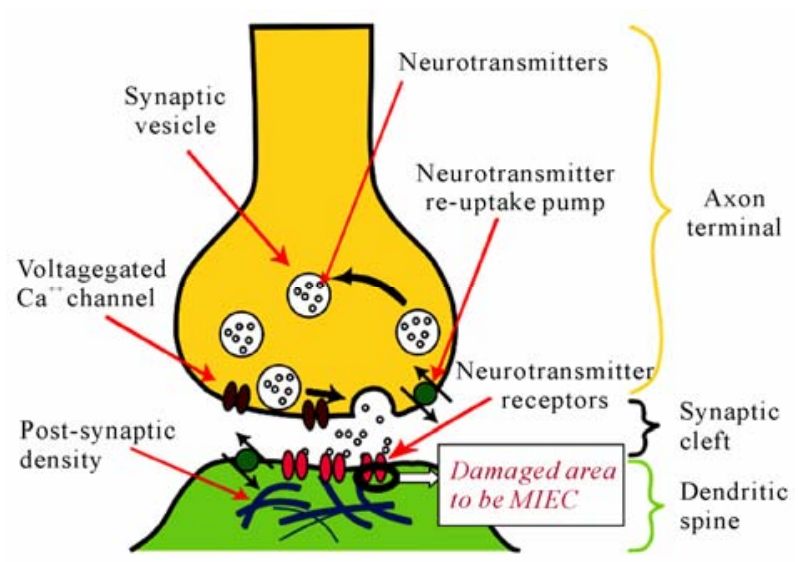

Figure 15. Without MIEC knowledge, it is impossible to notice the voltage down of neurotransmitter receptors. (From http://en.wikipedia.org/wiki/Major_depressive_disorder\#M onoamine_hypothesis).

But the classical thermodynamics is not completed still yet. Many mental diseases are caused from the illness of ion channels and many medicines are effective for ion channels. The differences between brain and SDC are shown in Table 2. The role of ionic activation energy is something internal dimensions. The fundamental meaning of the internal dimension is discussed [8]. The number of internal dimensions in theoretical physics is discussed empirically [9]. Internal dimensions in the brain are large enough to explain the consciousness, since there are numerous ion channels in the brain. The research of the consciousness should be in the area of electrochemistry. The fundamental thermo dynamical research of MIEC is needed.

\subsubsection{The Mechanism of Depression}

Depressive disorder is explained by monoamine hypothesis since 1980s. Using the present technology, the hypothesis cannot be confirmed still yet. When the detail mechanism of hypothesis will be clarified, the numerous people will be helped. The neurotransmitter receptors may be voltage down by the reduction of dense anode area where the parts were damaged to be MIEC.

\section{Conclusions}

Instead of the leakage current using Sm doped Ceria electrolytes in SOFCs, the constant voltage loss is proposed. Considering the separation of Boltzmann distribu tion and Maxwell's demon, the modifications of Wagner's equation are proposed. Wagner's equation is based on the Nernst-Plank equation. So, when the modifications are needed in Wagner's equation, the modifications of Nernst-Plank equation are needed. It means that the classical thermodynamics is not finished in the fundamental level. The effects of the modification are observed in MIEC. This topic is not an isolated and minor topic, but of vital importance to the scientific community.

\section{Acknowledgements}

This report is rewritten from the ECS transaction [7].

\section{REFERENCES}

[1] C. Wagner, "Beitrag zur Theorie des Anlaufvorgangs," Zeitschrift für Physikalische Chemie, Vol. B41, 1933, p. 42.

[2] H. Rickert, "Electrochemistry of Solids-An Introduction," Springer, Berlin, Heidelberg, 1982.

[3] T. Miyashita, "Necessity of Verification of Leakage Currents Using Sm Doped Ceria Electrolytes in SOFCs," Journal of Materials Science, Vol. 41, No. 10, 2006, pp. 3183-3184. doi:10.1007/s10853-006-6371-8

[4] T. Miyashita, "Current-Voltage Relationship Considering Electrode Degradation Using Sm-Doped Ceria Electrolytes in SOFCs," ECS Transactions, Vol. 35, 2011. (In Press).

[5] T. Miyashita, "The Limitations of Wagner's Equation in Solid-State Electrochemistry," ECS Transactions, Vol. 33, No. 32, 2011, pp. 11-18. doi:10.1149/1.3562554

[6] T. Miyashita, "Empirical Equation about Open Circuit Voltage in SOFC," Journal of Materials Science,Vol. 40, No. 22, 2005, p. 6027. doi:10.1007/s10853-005-4560-5

[7] T. Miyashita, "Fundamental Thermodynamic Modifications in Wagner's Equation in Solid State Electrochemistry," ECS Transactions, Vol. 28, 2010, pp. 39-49. doi:10.1149/1.3502443

[8] T. Miyashita, "Quantum Physics can be Understood in Terms of Classical Thermodynamics," Journal of Modern Physics, Vol. 2, 2011, pp. 26-29. doi:10.4236/jmp.2011.31005

[9] T. Miyashita, "Empirical Relations about the Number of Dimensions in Theoretical Physics with the Concept of Common and Unshared Dimensions," Journal of Modern Physics, Vol. 2, 2010, pp. 147-149. doi:10.4236/jmp.2010.12021 